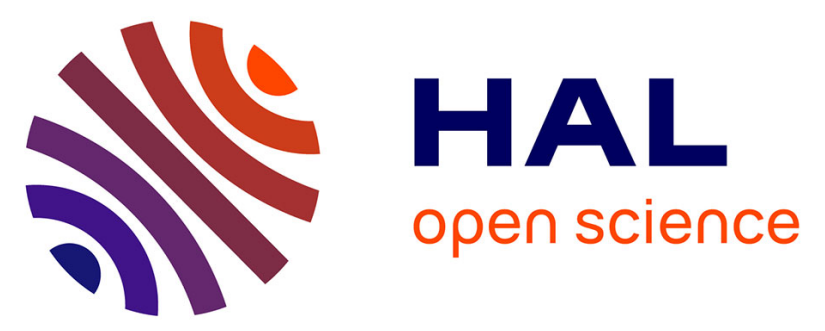

\title{
Single- and double-ionization processes using Gaussian-type orbitals: Benchmark on antiproton-helium collisions in the keV-energy range
}

J. W Gao, T. Miteva, Y. Wu, J. G Wang, A. Dubois, N. Sisourat

\section{- To cite this version:}

J. W Gao, T. Miteva, Y. Wu, J. G Wang, A. Dubois, et al.. Single- and double-ionization processes using Gaussian-type orbitals: Benchmark on antiproton-helium collisions in the keV-energy range. Physical Review A, 2021, 103 (3), 10.1103/PhysRevA.103.L030803 . hal-03185021

\section{HAL Id: hal-03185021 \\ https: / hal.sorbonne-universite.fr/hal-03185021}

Submitted on 30 Mar 2021

HAL is a multi-disciplinary open access archive for the deposit and dissemination of scientific research documents, whether they are published or not. The documents may come from teaching and research institutions in France or abroad, or from public or private research centers.
L'archive ouverte pluridisciplinaire HAL, est destinée au dépôt et à la diffusion de documents scientifiques de niveau recherche, publiés ou non, émanant des établissements d'enseignement et de recherche français ou étrangers, des laboratoires publics ou privés. 


\title{
Single and double ionization processes using Gaussian type orbitals: benchmark on antiproton-helium collisions in the keV energy range
}

\author{
J. W. Gao, ${ }^{1}$ T. Miteva,${ }^{2}$ Y. Wu,${ }^{3}$ J. G. Wang, ${ }^{3}$ A. Dubois,${ }^{2}$ and N. Sisourat ${ }^{2, *}$ \\ ${ }^{1}$ Key Laboratory of Radiation Physics and Technology of Ministry of Education, \\ Institute of Nuclear Science and Technology, \\ Sichuan University, Chengdu 610064, China \\ ${ }^{2}$ Sorbonne Université, CNRS, Laboratoire de Chimie \\ Physique - Matière et Rayonnement, F-75005 Paris, France \\ ${ }^{3}$ Institute of Applied Physics and Computational Mathematics, 100088, Beijing, China.
}

(Dated: March 29, 2021)

\begin{abstract}
We present a simple and efficient method for computing single and double ionization cross sections in ion-atom and ion-molecule collisions using $L^{2}$ Gaussian basis sets. Gaussian functions are widely employed to compute bound states of ions, atoms and molecules. However, the description of continuum states, and therefore ionization phenomena, remains a theoretical challenge. Our approach is tested on the benchmark system antiproton-helium collisions in the so-called intermediate energy range. A good agreement with numerically exact calculations is observed. The proposed method is general and can thus be employed in any collisional systems in the challenging non-perturbative regime. Our work opens the way to investigate multiple ionization processes by ion impact in multi-center poly-electronic systems.
\end{abstract}

\footnotetext{
*nicolas.sisourat@sorbonne-universite.fr
} 


\section{INTRODUCTION}

Since the pioneer work of Boys and coworkers [1] Gaussian functions are widely used in electronic structure calculations. Although they cannot reproduce the cusp at the nucleus of the true eigenstates [2], they provide a fairly good description of bound states and allow the efficient computation of integrals [3]. Gaussian functions, also called Gaussian type orbitals (GTOs), are therefore used in most quantum chemistry packages (see e.g. the special issue in $[4])$.

Because GTOs are square-integrable $L^{2}$ functions they cannot describe the exact boundary conditions of continuum states. Although there have been large efforts to find optimal GTOs to approximate these states [5-9] or to provide corrections to the GTOs finite boundary conditions (see e.g. [10-14]), it is still challenging to describe molecular states above the ionization thresholds.

An accurate description of continuum states is of particular relevance to describe ionization processes. In this work, we are interested in single and double ionization phenomena in ion-atom and ion-molecule collisions in the so-called intermediate energy range. In the context of these collisions, GTOs have been widely employed to describe bound-bound transitions (i.e. charge transfer and excitation) (see for example [15-21] and references therein). Ionization processes have also been considered but with less success. Using GTOs, the continuum states are approximated by the pseudo continuum states with energy above the ionization thresholds obtained by the diagonalization of the electronic Hamiltonian within the basis set. Single ionization cross sections have thus been rather accurately computed for small systems (mostly one-active electron atomic systems). Computing these cross sections for larger systems and considering multiple ionization processes remain, however, challenging and relevant tasks. In this work, we report on a simple and efficient approach for computing single and double ionization cross sections in ion-atom and ion-molecule collisions in the non-perturbative regime using GTOs. Using unsupervised machine learning classification techniques combined with pertinent properties of the pseudo continuum states, we sort these states into single and double electron continuum states. While we test this approach with the benchmark antiproton-helium collisional system, our proposed method can straightforwardly be applied to multicenter and polyelectronic systems.

The present paper is organized as follows. In the next section we outline the methods and 
computational details employed to compute the total and state-resolved single ionization and total double ionization cross sections. The results of the clustering analysis used to classify the pseudo continuum states into single and double electron continuum states are then reported (section III A). In section III B and III C, we present and discuss the single and double ionization cross sections in antiproton-helium collisions, respectively. They are followed by the conclusions in section IV. Atomic units are used throughout, unless explicitly indicated otherwise.

\section{METHODS AND COMPUTATIONAL DETAILS}

We use the well established straight-line impact parameter method (see $[22,23]$ and references therein) in which the nuclei follow classical trajectories while a quantum treatment describes the electronic dynamics. The position vector of the projectile relative to the target is $\vec{R}(t)=\vec{b}+\vec{v} t$ where $\vec{b}$ and $\vec{v}$ are the impact parameter and projectile velocity, respectively. For a two-electron system, the time-dependent electronic Schrödinger equation reads

$$
\left[H_{e}-i \frac{\partial}{\partial t}\right] \Psi\left(\vec{r}_{1}, \vec{r}_{2}, \vec{R}(t)\right)=0,
$$

where $H_{e}$ is the electronic Hamiltonian,

$$
H_{e}=\sum_{i=1,2}\left(-\frac{1}{2} \nabla_{i}^{2}+V_{T}\left(\vec{r}_{i}\right)+V_{P}\left(\vec{r}_{i}^{p}\right)\right)+\frac{1}{\left|\vec{r}_{1}-\vec{r}_{2}\right|},
$$

and $\vec{r}_{i}, \vec{r}_{i}^{p}=\vec{r}_{i}-\vec{R}(t)$ are the position vectors of the electrons with respect to the target and the projectile respectively.

In our work, the electronic wavefunction is written as

$$
\Psi\left(\vec{r}_{1}, \vec{r}_{2}, t\right)=\sum_{k=1}^{N} a_{k}(t) \chi_{k}\left(\vec{r}_{1}, \vec{r}_{2}\right) e^{-i E_{k} t}
$$

where $\chi_{k}\left(\vec{r}_{1}, \vec{r}_{2}\right)$ are singlet helium pseudo states and $E_{k}$ the corresponding energies. These states are obtained by diagonalization of the electronic Hamiltonian matrix which is built using Slater determinants of Hartree-Fock (HF) orbitals. All configurations (i.e. HF ground state, single and double excitations) are included. These full Configuration Interaction (CI) calculations are performed using the CIPPRES [24] plugin of the QP2 quantum chemistry package $[25,26]$.Note that in Eq. 3, a single generic index $k$ is used. However, it may refer to bound states as well as single and double electron pseudo continuum states (see below). 
The insertion of Eq. 3 into Eq. 1 results in a system of first-order coupled differential equations, written in matrix form as,

$$
i \frac{d}{d t} \mathbf{a}(t)=\mathbf{M}(\vec{b}, \vec{v}, t) \mathbf{a}(t)
$$

In the above equation, $\mathbf{a}(t)$ is the column vector of the time-dependent expansion coefficients and $\mathbf{M}$ is the coupling matrix. These equations are solved for a set of initial conditions (initial state $i, b$ and $v$ ) using the Adams-Bashforth-Moulton predictor-corrector integrator. The probabilities of the transition $i \rightarrow f$ for a given impact parameter and velocity read

$$
P_{i \rightarrow f}(b)=\lim _{t \rightarrow \infty}\left|a_{f}(t)\right|^{2} .
$$

The cross section for a given electronic process is then calculated as

$$
\sigma_{f}=2 \pi \int_{0}^{+\infty} b P_{i \rightarrow f}(b) d b .
$$

The cross sections for single and double ionization processes are obtained by plugging in the above equations (Eqs. 5 and 6 ) the expansion coefficients $a_{f}(t)$ (Eq. 3) corresponding to single and double electron pseudo continuum states. The procedure to separate these two kinds of states is discussed below.

In our computations, we used even-tempered Gaussian basis sets [27]: the Gaussian exponents $\alpha_{p}$ are expressed as a geometric sequence according to $\alpha_{p}=\alpha \beta^{1-p}(p=1,2, \ldots)$. Three different basis sets (namely 12s9p6d3f1g, 13s10p7d4f and 14s11p7d4f) have been used in order to evaluate the convergence of the cross sections. We report below the mean and standard deviation values of the set of cross sections obtained with these basis sets (the cross sections obtained with each individual basis set are given in the supplementary materials). The notation 12s9p6d for example indicates that 12 Gaussian functions s $(\ell=0), 9$ functions $\mathrm{p}(\ell=1)$ and 3 functions $\mathrm{d}(\ell=2)$ were included in the basis set. In our calculations, the lowest and largest values of $\alpha$ were fixed, respectively, at 0.001 and 400 for s functions, and 0.001 and 40 for higher angular momentum functions. The parameter $\beta$ was then chosen to reproduce the geometric sequence for a given number of functions. Depending on the basis set, between 9800 and 12000 configurations are thus used to describe the pseudo states. After full diagonalization of the Hamiltonian matrix, pseudo states with energy up to 5 a.u. are kept in the close-coupling expansion (i.e. between 3000 and 5000 states).

The pseudo states of helium can be grouped into three classes: bound states which describe elastic and excitation processes, single and double electron pseudo continuum states 
used to model single and double ionization phenomena, respectively. The first class of states have energy below the lowest ionization potential of the system (i.e. -2.0 a.u. for helium) and can thus be clearly identified. States with higher energies are pseudo continuum states because Gaussian functions do not have the correct outgoing-wave boundary conditions. Pseudo states with energy between the lowest ionization threshold and the double ionization one can be considered as single electron continuum states. Note that for these pseudo states, the cationic states of the remaining bound electron cannot be clearly defined because it should involve the true continuum of the cationic channels. Furthermore, above the double ionization threshold a distinction between single and double electron continuum states cannot be rigorously made. We propose below a ad hoc procedure to i) obtain the states of the remaining cation after single ionization, allowing thus to compute state-resolved excitation-ionization cross sections and ii) to separate single and double electron continuum states.

To sort the pseudo continuum states into single and double electron continuum states, one needs to find some properties, so-called features below, that are different for the two kind of states. The choice of suitable features is a difficult task of any machine learning algorithm and has thus fed the literature for the last two decades [28]. In this work, we use two features for each pseudo continuum state: the sum of the norm of the Dyson orbitals and the electron-electron repulsion energy. Hereafter, we use the notation SND for the former and EER for the latter quantity. The SND for the state $k$ is defined as

$$
\mathrm{SND}_{\mathrm{k}}=\sum_{j} \int d \vec{r}\left|\phi_{k j}(\vec{r})\right|^{2} .
$$

In this equation, $\phi_{k j}(\vec{r})$ are the so-called Dyson orbitals,

$$
\phi_{k j}(\vec{r})=\int d \vec{r}_{2} \chi_{j}^{H e^{+}}\left(\vec{r}_{2}\right) \chi_{k}^{H e}\left(\vec{r}_{1}, \vec{r}_{2}\right)
$$

and the sum $j$ is performed over all bound states of $\mathrm{He}^{+}$. The EER for the state $k$ is computed as

$$
\mathrm{EER}_{\mathrm{k}}=\int d \vec{r}_{1} d \vec{r}_{2} \chi_{k}^{H e}\left(\vec{r}_{1}, \vec{r}_{2}\right) \frac{1}{\left|\vec{r}_{1}-\vec{r}_{2}\right|} \chi_{k}^{H e}\left(\vec{r}_{1}, \vec{r}_{2}\right)
$$

While there is no rigourous justification for the use of these features, our results suggest that they are relevant to sort the pseudo continuum states. Furthermore, their relevance in sorting the states may be rationalized as follows: the SND of a true single electron continuum 
state should be one since one electron remains bound after the ionization process while for a true double electron continuum state the SND should go to zero. The EER of both kinds

of true continuum states should be close to zero and this feature should not be relevant. However, as shown and discussed below, in a finite basis set the EER of the double electron pseudo continuum states are generally larger that those of single electron pseudo continuum states. The use of the EER is therefore pertinent.

Using these features, we have performed a clustering analysis (see III A) to group the pseudo continuum states into two "clusters" corresponding to single and double electron continuum states. K-means [29] and spectral clustering [30] approaches, which are two wellestablished methods for unsupervised machine learning classification, have been employed. Same clusters are obtained with both methods and only results from the K-means clustering approach are reported below. These analysis have been done with the Scikit-learn package [31].

The partial single ionization cross sections $\tilde{\sigma}_{j}$ (i.e. after the collision $\mathrm{He}^{+}$is in the ground or excited state $j$ ) are obtained as:

$$
\tilde{\sigma}_{j}=\sum_{k} \frac{P_{k j}}{\sum_{j} P_{k j}} \sigma_{k}
$$

where $\sigma_{k}$ is the cross section of the pseudo continuum state $k$ (classified as a single continuum state in the clustering analysis) and $P_{k j}$ is the norm of the Dyson orbital $\phi_{k j}$. The total double ionization cross sections are the sum of the cross sections of the pseudo continuum states belonging to the double electron continuum cluster.

\section{RESULTS AND DISCUSSIONS}

\section{A. Single versus double electron continuum states}

Before reporting the single and double ionization cross sections in antiproton helium collisions, we present the results of our approach to separate single and double electron continuum states. The energy of the helium pseudo states obtained with the 12s9p6d3f1g basis set are shown in Fig. 1. There are 43 bound states, 1351 states whose energies are between -2.0 and 0.0 a.u. (i.e. assigned to single electron continuum states) and 989 states

with energies above the double ionization threshold. In order to illustrate the relevance of 
the SND and the EER as features for the clustering analysis, we plot them for every state in Fig. 2: the bound states exhibit a SND close to 1 since the helium cation states (within the basis set) represent a complete basis for the helium states. Except for the ground state of helium, the EER of the bound states are small. The SND for the higher states with energy below the double ionization threshold are also close to one. However, as the energy of the states rises the SND decreases owing to the finite basis set. The states with energy above 0 a.u. are the most interesting ones for our analysis. In order to separate these states into two groups (i.e. single and double electron continuum states) we applied the K-means clustering analysis as implemented in the Scikit-learn package. The results are shown in Fig. 3: single electron continuum states present in general a SND close to 0.5 and small EER, while the SND for the double electron continuum states are near zero and the EER are larger. The latter observation might be counter-intuitive since two electrons in a real continuum should have nearly zero electron-electron repulsion. However, owing to the finite character of our basis set, the two electrons in the pseudo continuum are actually spatially closer than when one electron is bound (i.e. close to the nuclei) and one is "free". Using the results of this classification and the ad hoc procedure presented above, we compute the state-resolved single ionization and the double ionization cross sections. Results are shown and discussed hereafter.

The antiproton-helium collisional system is a prototype and has thus been widely investigated [32-40] (see also [41, 42] for recent reviews on antiproton collisions and [37] for a detailed discussion on electron-electron correlation effects in the antiproton-helium system). For this two-electron system, a full numerical solution can be performed as in [38]. In the following, we use the results of [38] as references to compare with the total cross sections computed within our $L^{2}$ Gaussian basis set approach. Total single and double ionization cross sections have also been measured, and computed with different approaches. The results of [38] agree well with the available measurements and a detailed comparison can be found in [38]. To our knowledge, state-resolved single ionization cross sections have only been reported in [43] and references therein. Note that these cross sections were computed with perturbative approaches. We use the results of $[43,44]$ below for comparison. 


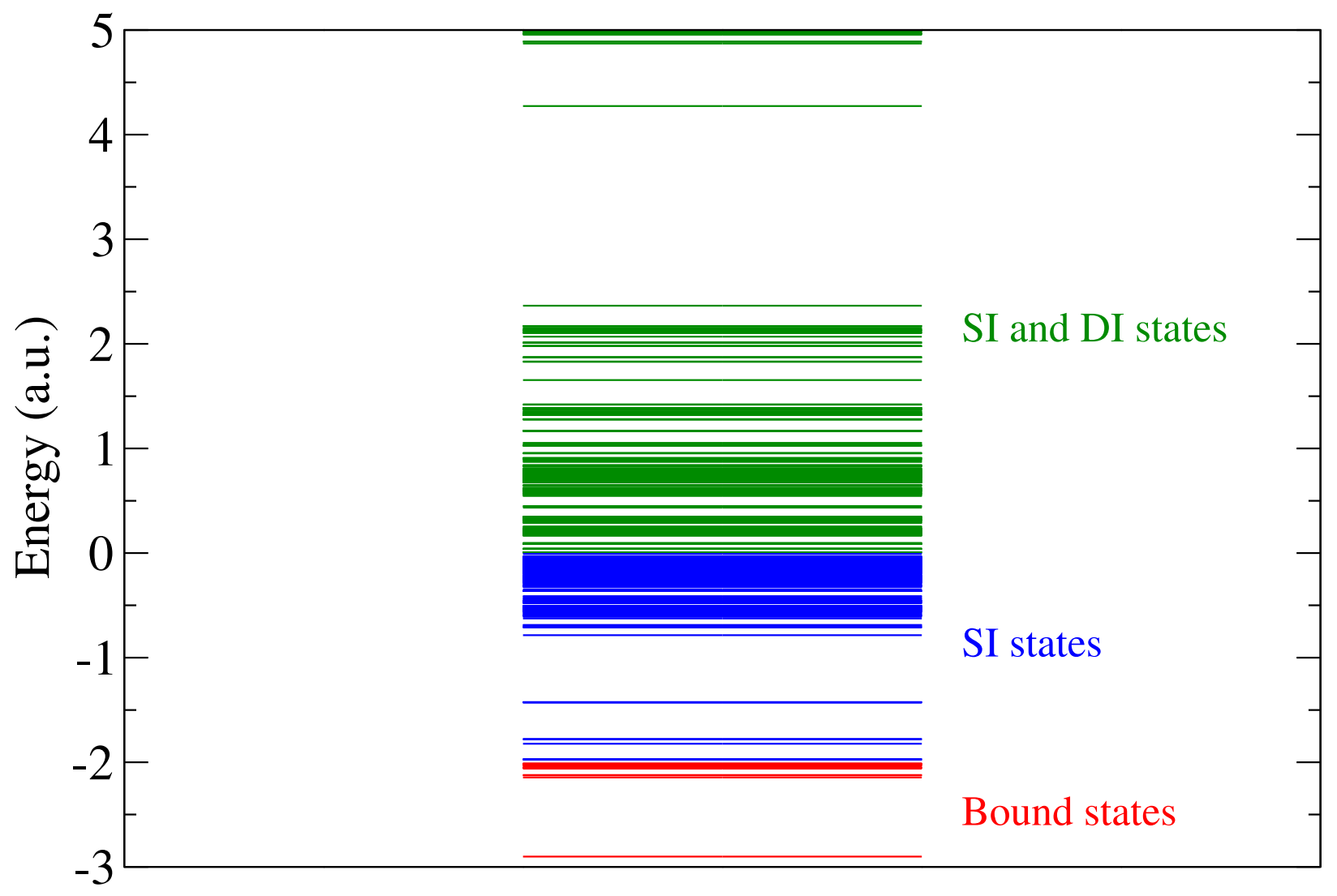

FIG. 1. The Helium pseudo energies obtained with the 12s9p6d3f1g basis set.

\section{B. Single ionization cross sections}

The mean total and state-resolved single ionization cross sections computed with our three basis sets are shown in Fig. 4. The total cross sections thus obtained agree well with the reference data of [38]. The corresponding standard deviations are fairly small, showing a good convergence of the total cross sections with respect to the basis set. Our state-resolved single ionization cross sections are in general larger than those reported in [43], especially for the cross sections of ionization-excitation into $\mathrm{He}^{+}(2 p)$ (i.e. about a factor of 3 at the lowest overlapping energy and up to an order of magnitude at higher energies). We mention that in [43], the authors have employed the same perturbative method to compute the state-resolved single ionization cross sections for proton and electron impact too. The cross sections they obtained are overall smaller than the experimental ones (see [43] for a detailed discussion on the possible reasons for such disagreement). The perturbative approach may therefore underestimate the cross sections for antiproton collisions in the energy domain 


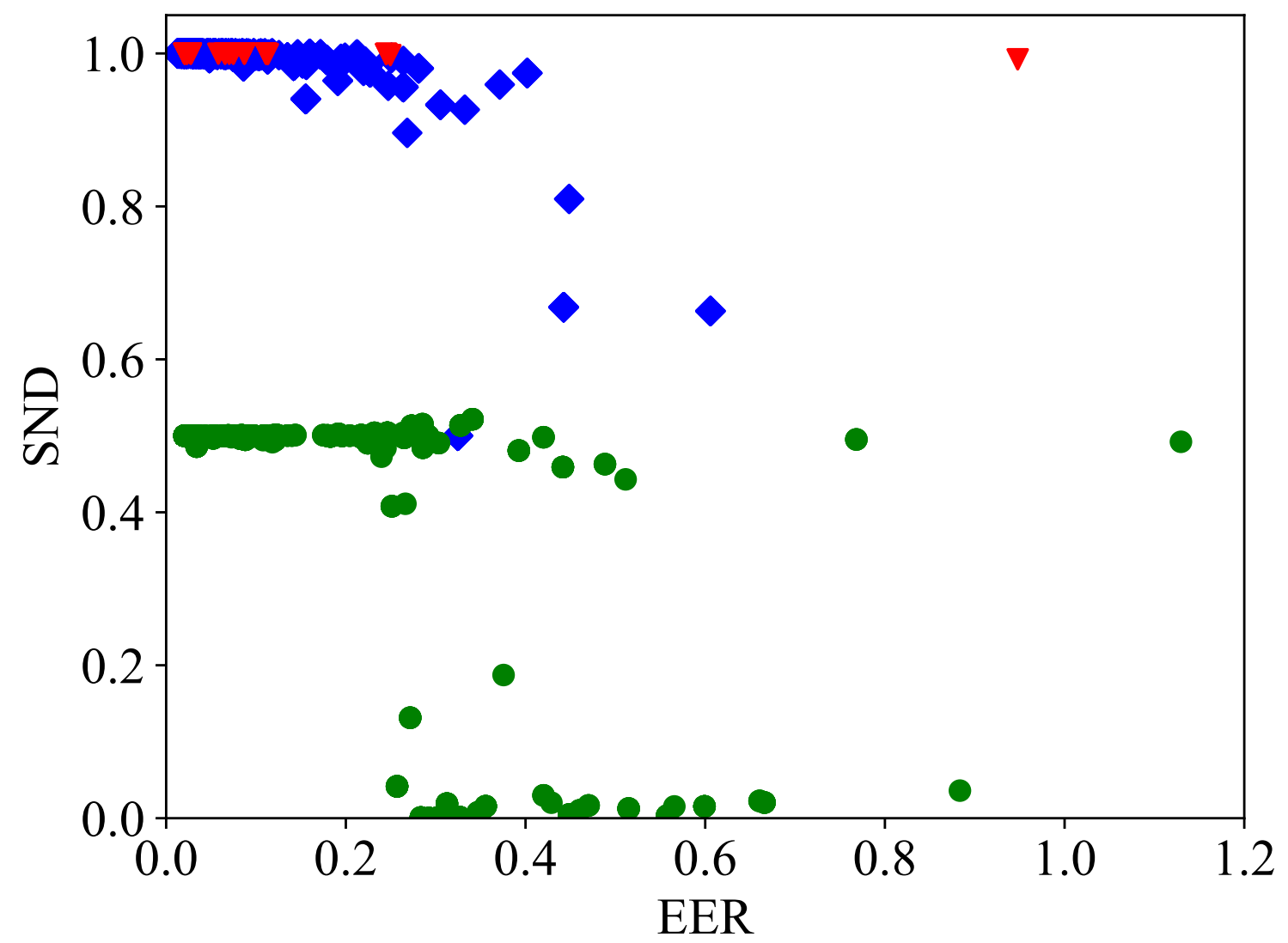

FIG. 2. Sum of the norm of the Dyson orbitals (SND) plotted as a function of the electron-electron repulsion energy (EER) for every Helium states: red triangles are used for states with energy below the lowest single ionization potential, blue diamonds represent the states with energy between -2.0 and 0.0 a.u. and green dots show the features for states with positive energy. These quantities are reported in atomic units.

under consideration. On the other hand, owing to the use of pseudo continuum states in our calculations the electronic correlation in the ionization-excitation processes may be too large leading to an overestimation of the cross sections. Further experimental and theoretical works are needed to conclude. 


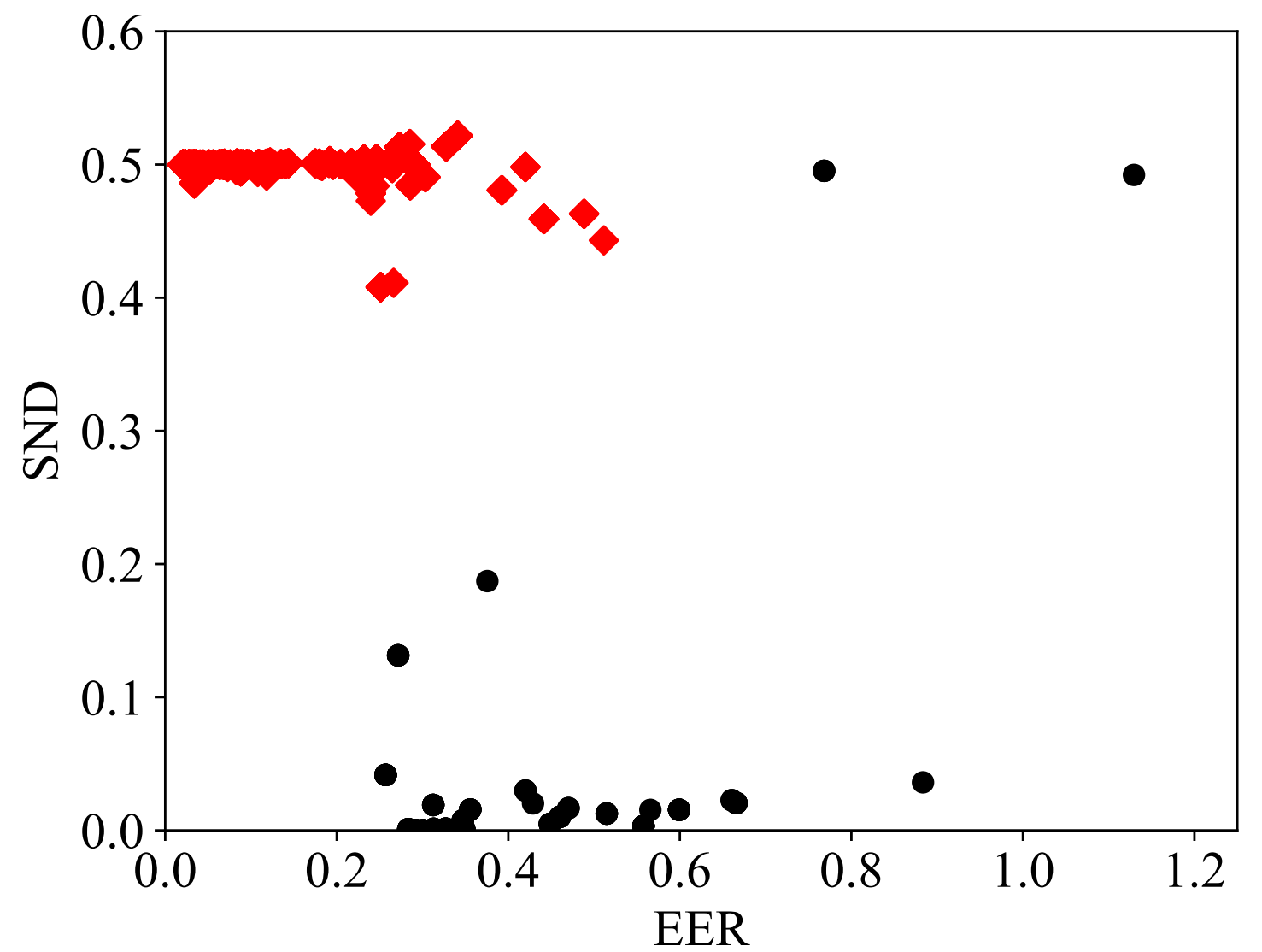

FIG. 3. Sum of the norm of the Dyson orbitals (SND) plotted as a function of the electron-electron repulsion energy (EER) for Helium states with positive energy: those identified as single and double electron continuum states using the K-means clustering analysis are shown in red diamonds and black dots, respectively. These quantities are reported in atomic units.

\section{Double ionization cross sections}

The total double ionization cross sections computed with our approach are shown with the reference data of [38] in Fig. 5. For comparison, we report the cross sections obtained after the clustering analysis (labeled as "From analysis" in the figure) and the sum of the cross sections over all pseudo continuum states of positive energies ("Upper limit" in the figure). The latter represent an upper limit of the double ionization cross sections. The difference between the "Upper limit" and "From analysis" cross sections thus represent the single ionization cross sections of states having positive energies. Without the clustering analysis, the double ionization cross sections are far too large because part of the single 


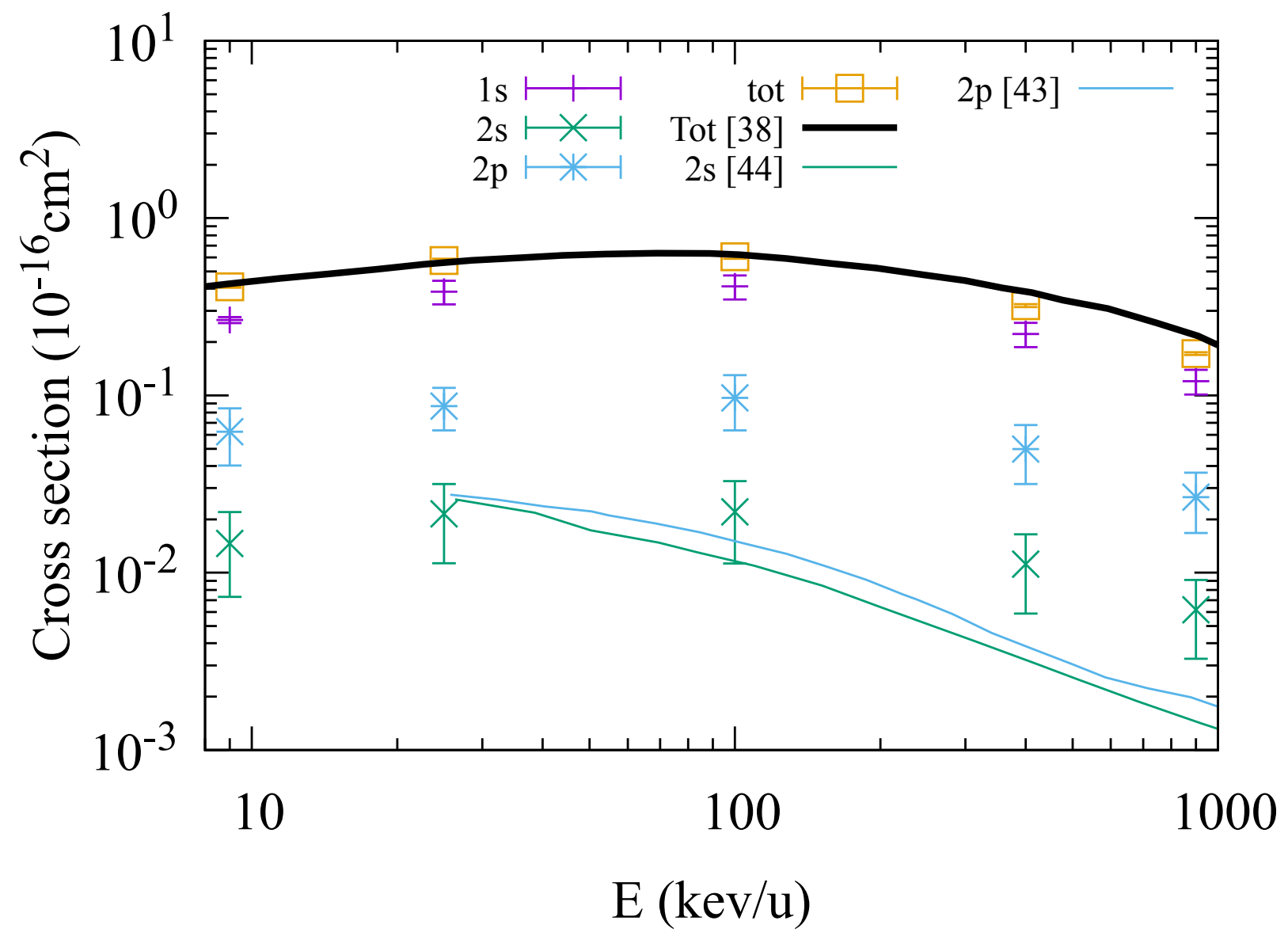

FIG. 4. Total and state-resolved single ionization cross sections. The reference calculations of [38] and of $[43,44]$ are shown in full lines.

ionization cross sections are included. Furthermore, these upper limits depend strongly on the basis sets as shown by the large standard deviations. The cross sections obtained after the clustering analysis agree well with the reference results and depend less than the upper limits on the basis sets. It is therefore clear that the clustering analysis is crucial to obtain accurate double ionization cross sections.

\section{CONCLUSIONS}

In this work, we have theoretically investigated single and double ionization processes in antiproton-helium collisions in the non-perturbative regime. The impact parameter method and a close-coupling approach using Gaussian-type orbitals are employed. Gaussian functions are widely employed to compute bound states of ions, atoms and molecules. However, 


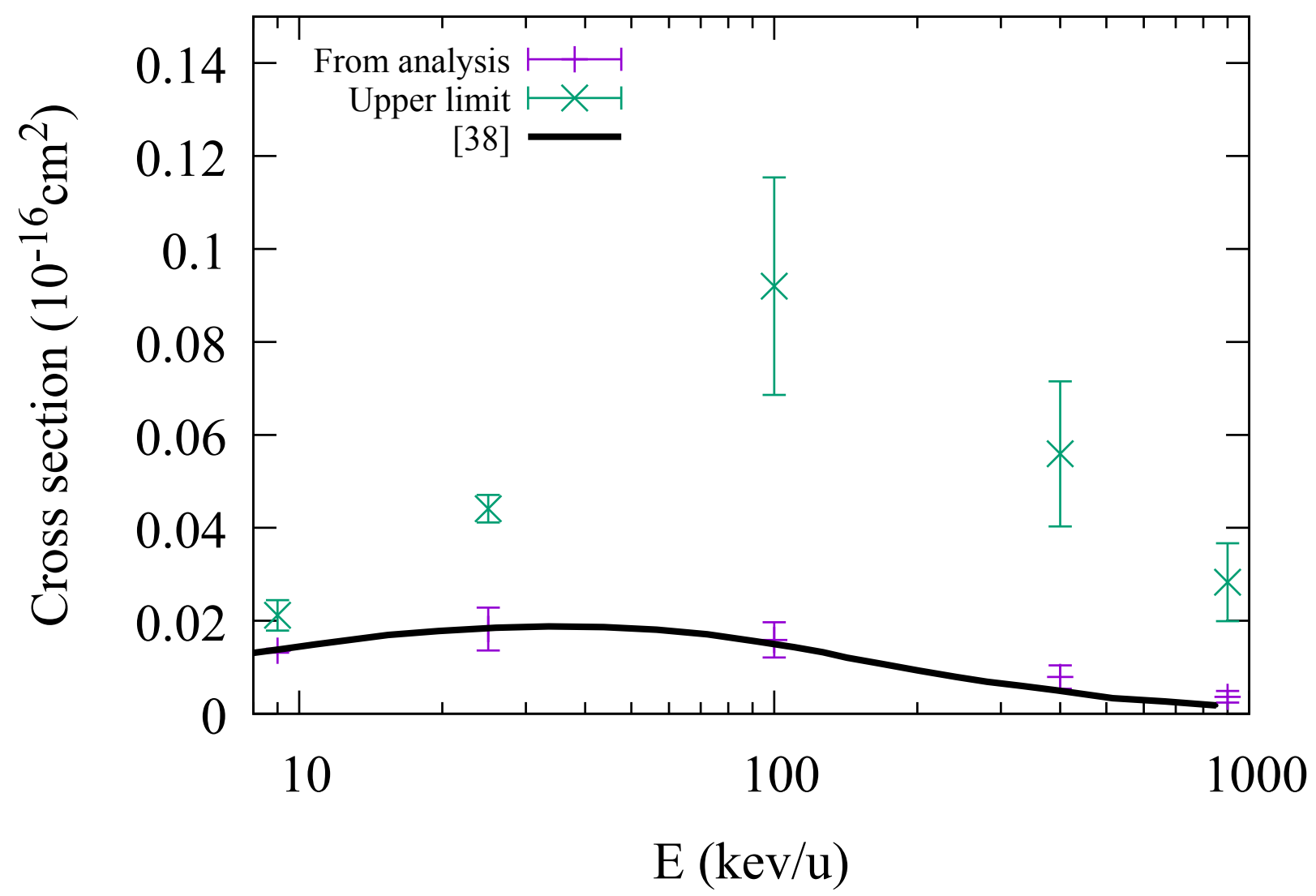

FIG. 5. Double ionization cross sections. The cross sections obtained after the clustering analysis are labeled "From analysis" while the sum of the cross sections over all pseudo continuum states of positive energies are marked "Upper limit". The reference calculations of [38] are shown in full black line.

the description of continuum states with such $L^{2}$ functions, and therefore ionization phenomena, remain a theoretical challenge. A simple and efficient method for computing single and double ionization cross sections in ion-atom and ion-molecule collisions using these basis sets is reported and tested. Good agreement with available reference data is observed.

Our proposed method has been tested on collisions with antiproton but it can straightforwardly be applied to other collision systems. The clustering analysis method reported here is used to sort the pseudo continuum states into single and double electron continuum states and can be employed to any systems. In many relevant ion-atom and ion-molecule collisions, electron transfer can take place, which is not the case with the antiproton. In our close-coupling expansion, the electron transfer channels can be accounted for thanks 
to the pseudo continuum states. As in any truncated expansion, convergence of the cross sections with respect to the set of states must however be checked. Providing that convergence is reached the overall approach presented here is general. Our results thus open the way to investigate multiple ionization processes by ion impact in multi-center poly-electronic systems.

[1] S. F. Boys and A. C. Egerton, Proc. R. Soc. Lond. A 200, 542 (1950).

[2] W. Klopper and W. Kutzelnigg, J. Mol. Struct.: THEOCHEM 135, 339 (1986).

[3] S. Reine, T. Helgaker, and R. Lindh, WIREs Comput. Mol. Sci. 2, 290 (2012).

[4] C. D. Sherrill, D. E. Manolopoulos, T. J. Martínez, and A. Michaelides, J. Chem. Phys. 153, $070401(2020)$.

[5] K. Kaufmann, W. Baumeister, and M. Jungen, J. Phys. B 22, 2223 (1989).

[6] A. Faure, J. Gorfinkiel, L. Morgan, and J. Tennyson, Comput. Phys. Commun. 144, 224 (2002).

[7] S. Klinkusch, P. Saalfrank, and T. Klamroth, J. Chem. Phys. 131, 114304 (2009).

[8] E. Coccia, B. Mussard, M. Labeye, J. Caillat, R. Taïeb, J. Toulouse, and E. Luppi, Int. J. Quantum Chem. 116, 1120 (2016).

[9] A. P. Woźniak, M. Lesiuk, D. K. Efimov, M. Mandrysz, J. S. Prauzner-Bechcicki, M. Ciappina, E. Pisanty, J. Zakrzewski, M. Lewenstein, and R. Moszyński, "A systematic construction of gaussian basis sets for the description of laser field ionization and high-harmonic generation," (2020), arXiv:2007.10375 [physics.chem-ph].

[10] U. V. Riss and H.-D. Meyer, J. Phys. B 26, 4503 (1993).

[11] N. Moiseyev, J. Phys. B 31, 1431 (1998).

[12] P. Langhoff, Chem. Phys. Lett. 22, 60 (1973).

[13] F. Müller-Plathe and G. H. F. Diercksen, Phys. Rev. A 40, 696 (1989).

[14] E. Coccia, R. Assaraf, E. Luppi, and J. Toulouse, J. Chem. Phys. 147, 014106 (2017).

[15] W. Fritsch and C. D. Lin, Phys. Rep. 202, 1 (1991).

[16] R. Hildenbrand, N. Grun, and W. Scheid, J. Phys. B 28, 4781 (1995).

[17] N. Toshima, Phys. Rev. A 59, 1981 (1999).

[18] M. C. Bacchus-Montabonel, M. Łabuda, Y. S. Tergiman, and J. E. Sienkiewicz, Phys. Rev. 
A 72, 052706 (2005).

[19] N. Sisourat, I. Pilskog, and A. Dubois, Phys. Rev. A 84, 052722 (2011).

[20] P. M. M. Gabás, L. F. Errea, L. Méndez, and I. Rabadán, Phys. Rev. A 85, 012702 (2012).

[21] J. W. Gao, Y. Wu, J. G. Wang, A. Dubois, and N. Sisourat, Phys. Rev. Lett. 122, 093402 (2019).

[22] D. R. Bates and R. McCarroll, Proc. R. Soc. Lond. A 245, 175 (1958).

[23] B. H. Bransden and M. R. C. McDowell, Charge exchange and the theory of ion-atom collisions (Clarendon Press, 1992).

[24] N. Sisourat, "CIPPRES," Available: https://github.com/sisourat/qp2_plugins_ nsisourat, (2020).

[25] Y. Garniron, T. Applencourt, K. Gasperich, A. Benali, A. Ferté, J. Paquier, B. Pradines, R. Assaraf, P. Reinhardt, J. Toulouse, P. Barbaresco, N. Renon, G. David, J.-P. Malrieu, M. Véril, M. Caffarel, P.-F. Loos, E. Giner, and A. Scemama, J. Chem. Theory Comput. 15, 3591 (2019).

[26] See: https://quantum-package.readthedocs.io/en/master/.

[27] I. Cherkes, S. Klaiman, and N. Moiseyev, Int. J. Quantum Chem. 109, 2996 (2009).

[28] J. Behler, J. Chem. Phys. 145, 170901 (2016).

[29] D. Arthur and S. Vassilvitskii, in Proceedings of the Twenty-Second Annual Symposium on Computational Geometry, SCG '06 (Association for Computing Machinery, New York, NY, USA, 2006) p. 144-153.

[30] U. V. Luxburg, "A tutorial on spectral clustering," (2007), arXiv:0711.0189 [cs.DS].

[31] F. Pedregosa, G. Varoquaux, A. Gramfort, V. Michel, B. Thirion, O. Grisel, M. Blondel, P. Prettenhofer, R. Weiss, V. Dubourg, J. Vanderplas, A. Passos, D. Cournapeau, M. Brucher, M. Perrot, and E. Duchesnay, J. Mach. Learn. Technol. 12, 2825 (2011).

[32] L. H. Andersen, P. Hvelplund, H. Knudsen, S. P. Mo/ller, J. O. P. Pedersen, S. Tang-Petersen, E. Uggerho/j, K. Elsener, and E. Morenzoni, Phys. Rev. A 41, 6536 (1990).

[33] P. Hvelplund, H. Knudsen, U. Mikkelsen, E. Morenzoni, S. P. Moller, E. Uggerhoj, and T. Worm, J. Phys. B 27, 925 (1994).

[34] H. Knudsen, H.-P. E. Kristiansen, H. D. Thomsen, U. I. Uggerhøj, T. Ichioka, S. P. Møller, C. A. Hunniford, R. W. McCullough, M. Charlton, N. Kuroda, Y. Nagata, H. A. Torii, Y. Yamazaki, H. Imao, H. H. Andersen, and K. Tökesi, Phys. Rev. Lett. 101, 043201 (2008). 
[35] X. Guan and K. Bartschat, Phys. Rev. Lett. 103, 213201 (2009).

[36] H. Knudsen, H.-P. Kristiansen, H. Thomsen, U. Uggerhøj, T. Ichioka, S. Møller, C. Hunniford, R. McCullough, M. Charlton, N. Kuroda, Y. Nagata, H. Torii, Y. Yamazaki, H. Imao, H. Andersen, and K. Tökesi, Nucl. Instrum. Methods Phys. Res., B 267, 244 (2009).

[37] M. Baxter and T. Kirchner, Phys. Rev. A 87, 062507 (2013).

[38] S. Borbély, J. Feist, K. Tőkési, S. Nagele, L. Nagy, and J. Burgdörfer, Phys. Rev. A 90, 052706 (2014).

[39] I. B. Abdurakhmanov, A. S. Kadyrov, I. Bray, and K. Bartschat, Phys. Rev. A 96, 022702 (2017).

[40] S. Borbély, X.-M. Tong, S. Nagele, J. Feist, I. Březinová, F. Lackner, L. Nagy, K. Tőkési, and J. Burgdörfer, Phys. Rev. A 98, 012707 (2018).

[41] T. Kirchner and H. Knudsen, J. Phys. B 44, 122001 (2011).

[42] M. Schulz, Ion-Atom Collisions: The Few-Body Problem in Dynamic Systems (De Gruyter, Berlin/Boston, 2019).

[43] L. Nagy and Á. Benedek, J. Phys. B 35, 491 (2002).

[44] J. Mezei and L. Nagy, Nucl. Instrum. Methods Phys. Res., B 154, 295 (1999). 